\title{
Switchable Charge Injection Barrier in an Organic Supramolecular Semiconductor
}

\author{
Andrey V. Gorbunov, Andreas T. Haedler, Tristan Putzeys, R. Helen Zha, Hans-Werner \\ Schmidt, Milan Kivala, Indre Urbanaviciute, Michael Wubbenhorst, E. W. Meijer and \\ Martijn Kemerink
}

\section{Linköping University Post Print}

\section{Tweet}

N.B.: When citing this work, cite the original article.

Original Publication:

Andrey V. Gorbunov, Andreas T. Haedler, Tristan Putzeys, R. Helen Zha, Hans-Werner Schmidt, Milan Kivala, Indre Urbanaviciute, Michael Wubbenhorst, E. W. Meijer and Martijn Kemerink, Switchable Charge Injection Barrier in an Organic Supramolecular Semiconductor, 2016, ACS Applied Materials and Interfaces, (8), 24, 15535-15542.

http://dx.doi.org/10.1021/acsami.6b02988

Copyright: American Chemical Society

http://pubs.acs.org/

Postprint available at: Linköping University Electronic Press

http://urn.kb.se/resolve?urn=urn:nbn:se:liu:diva-130278 


\section{Switchable Charge Injection Barrier in an Organic}

\section{Supramolecular Semiconductor}

Andrey V. Gorbunov ${ }^{1}$, Andreas T Haedler ${ }^{2}$, Tristan Putzeys ${ }^{3}$, R. Helen Zha ${ }^{2}$, Hans-Werner

Schmidt ${ }^{4}$, Milan Kivala ${ }^{5}$, Indre Urbanavičiūtè ${ }^{6}$, Michael Wübbenhorst ${ }^{3}$, E. W. Meijer ${ }^{2}$, Martijn Kemerink ${ }^{*}, 1,6$

${ }^{1}$ Department of Applied Physics, Eindhoven University of Technology, PO Box 513, 5600 MB Eindhoven, The Netherlands

${ }^{2}$ Institute of Complex Molecular Systems, Laboratory of Macromolecular and Organic Chemistry, Eindhoven University of Technology, P.O. Box 513, 5600 MB, Eindhoven, The Netherlands

${ }^{3}$ Department of Physics and Astronomy, Laboratory for Soft Matter and Biophysics, KU Leuven, Celestijnenlaan 200D, B-3001 Heverlee, Belgium

${ }^{4}$ Makromolekuläre Chemie I, Bayreuther Institut für Makromolekülforschung (BIMF), and Bayreuther Zentrum für Kolloide und Grenzflächen (BZKG), Universität Bayreuth, 95440 Bayreuth, Germany

${ }^{5}$ Lehrstuhl für Organische Chemie I, Department Chemie und Pharmazie, FriedrichAlexander-Universität Erlangen-Nürnberg, 91054 Erlangen, Germany

${ }^{6}$ Complex Materials and Devices, Department of Physics, Chemistry and Biology (IFM), Linköping University, 58183 Linköping, Sweden

Keywords: organic semiconductors; current switching; memories; polarization; rectification; dipolar switching; injection barrier 


\section{ABSTRACT}

We disclose a supramolecular material which combines semiconducting and dipolar functionalities. The material consists of a discotic semiconducting carbonyl-bridged triarylamine core which is surrounded by three dipolar amide groups. In thin films, the material self-organizes in a hexagonal columnar fashion through $\pi$-stacking of the molecular core and hydrogen bonding between the amide groups. Alignment by an electrical field in a simple metal/semiconductor/metal geometry induces a polar order in the interface layers near the metal contacts that can be reversibly switched, while the bulk material remains non-polarized. On suitably chosen electrodes, the presence of an interfacial polarization field leads to a modulation of the barrier for charge injection into the semiconductor. Consequently, a reversible switching is possible between a high-resistance, injection-limited off-state and a low-resistance, space-charge-limited on-state. The resulting memory diode shows switchable rectification with on/off ratios of up to two orders of magnitude. This demonstrated multifunctionality of a single material is a promising concept towards possible application in lowcost, large-area, non-volatile organic memories. 


\section{INTRODUCTION}

Many years of research have been used to search for the elusive immortal organic memory. ${ }^{1,2}$ It is desired because of its re-writability, non-volatility, non-destructive electrical readout and scalability. The latter requires compatibility with a crossbar array to yield the highest information density with the simplest possible design. ${ }^{3}$ As a consequence the memory element must be rectifying and have a switchable conductivity. To this date, the most viable candidate system that fulfills these criteria is the so-called MemOLED, introduced by Asadi et al. ${ }^{4,5}$ Key to its design is that the bistable memory functionality is introduced by an organic ferroelectric polymer, poly[(vinylidenefluoride-co-trifluoroethylene] (P[VDF-TrFE]), while the conductive readout and rectification functionalities are introduced by an organic semiconductor polymer. Blending the two polymers leads, under proper processing conditions, ${ }^{6}$ to a phase separated blend consisting of semiconductor domains embedded in a ferroelectric matrix. ${ }^{7}$ When sandwiched as an active layer between suitably chosen metal electrodes, the polarization charge on the ferroelectric material modulates the barrier for charge injection into the semiconductor, leading to a rectifying and switchable device conductivity. ${ }^{8,9}$ Many advantages of this strategy, including compatibility with the crossbar architecture, ${ }^{10,11}$ are giving important new avenues to the actual implementation into active organic/polymeric devices. Following these important steps using phase-separated blends, some intriguing challenges remain. Is it possible to create a larger device area for the modulation of the injection barrier, as it is now limited to the narrow rim at the perimeter of the semiconducting domain. ${ }^{[12}$ The fact that the switching is not a bulk effect also constrains the minimum feature size to the typical micrometer length scale of the spontaneous $^{12}$ or lithographically-controlled ${ }^{13-15}$ phase separation. Finally, the polarization of the ferroelectric in the critical region around the semiconducting domains requires a higher stability to not fundamentally limit data retention. ${ }^{16}$ Hence, it could be argued that there is a clear need for a single material in which the dipolar and semiconducting properties are 
combined to yield a similar operational mechanism, while avoiding the difficulties associated with using a phase separated blend.

Here, we demonstrate an organic memory diode with a bistable, rectifying conductivity with an on/off ratio of two orders of magnitude. The heart of the device is a single molecular compound, consisting of a $\pi$-conjugated semiconducting core surrounded by three dipolar amide groups. In thin films, the material organizes into a hexagonal columnar packing, facilitating both effective charge transport and collective dipolar behavior. Although the bulk of the material is found to remain unpoled, dipolar switching in the interfacial areas leads to an effective modulation of the charge injection barrier and thereby to conductivity switching. 


\section{RESULTS AND DISCUSSION}

$a-$ molecular design and optical characterization of thin films

The investigated molecule, a-CBT, is shown in Fig 1(a) and consists of a planar carbonylbridged triarylamine core with three amide groups attached in 2, 6, and 10 positions. Attached to this CBT trisamide are three 3,4,5-tri(octyloxy)benzenes to enhance solubility and facilitate processing. The synthesis of the molecule is described in the supporting information where also its full name is given. The synthesis follows a general preparation methodology, which is reported elsewhere. ${ }^{17}$

For the current application, the $\pi$-conjugated semiconducting core enables charge transport ${ }^{18}$ whereas the peripheral amide groups provide bi-stable dipolar switching. ${ }^{19}$ The latter effect can result from a collective re-orientation of head-to-tail stacked micro-dipoles. ${ }^{20}$ Both efficient charge transport and dipolar switching require significant long-range order, which is promoted by the $\pi$-stacking of the planar, aromatic carbonyl-bridged triarylamine core and three-fold symmetric hydrogen bonding of the adjacent amide groups. Previous research has shown that this combination of supramolecular motifs leads to the formation of strongly coupling $\mathrm{H}$ aggregated CBT units along the columnar stacks. ${ }^{21}$ 

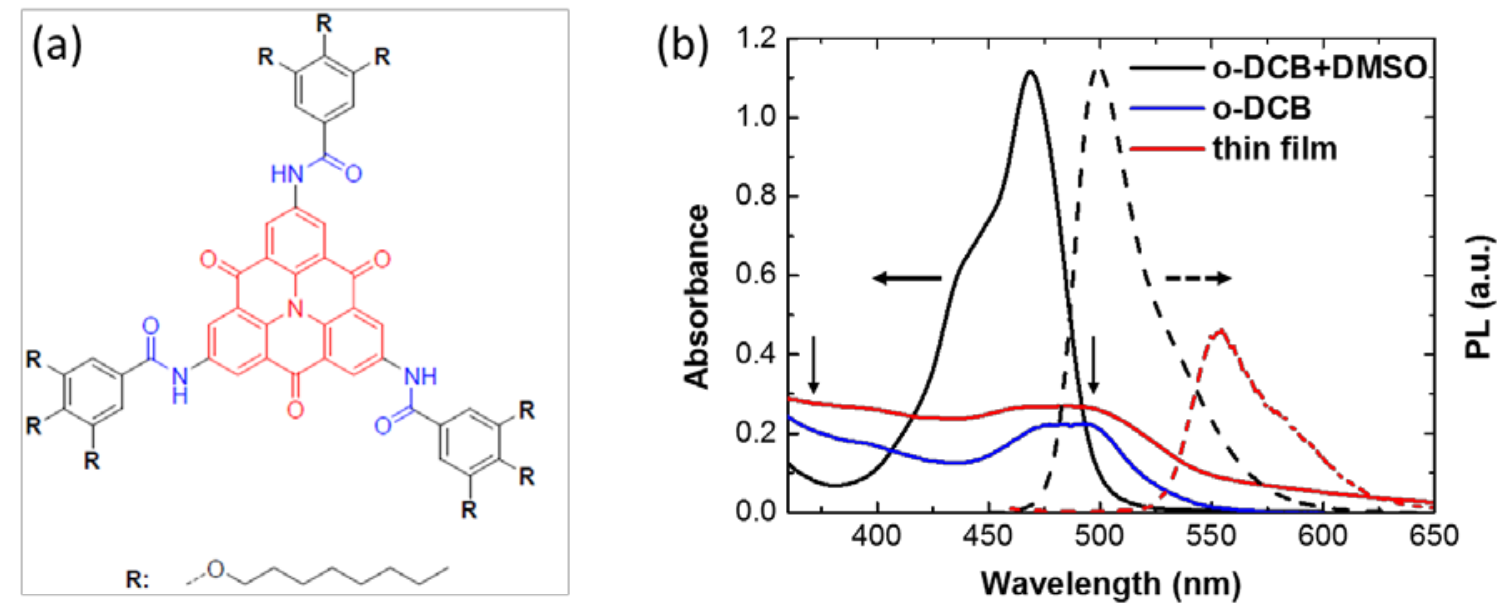

Fig. 1. (a) Molecular structure of a-CBT. $\mathrm{R}=\mathrm{OC}_{8} \mathrm{H}_{17}$. (b) $\mathrm{UV}$-Vis absorption (solid lines) and photoluminescence (PL) (dashed lines) spectra of a-CBT in solution $\left(5 \cdot 10^{-5} \mathrm{M}\right.$, black and blue lines) and in thin film (red lines).

In ortho-dichlorobenzene (o-DCB) solution $\left(5 \cdot 10^{-5} \mathrm{M}\right)$ with the addition of a drop of DMSO, a-CBT is molecularly dissolved. In this state, the absorption and fluorescence spectra (black solid and dashed lines in Fig. 1(b)) are mirror images with a small Stokes shift and peak maxima at $470 \mathrm{~nm}$ and $500 \mathrm{~nm}$, respectively. The absorption spectrum of a-CBT in pure o-DCB $\left(5 \cdot 10^{-}\right.$ ${ }^{5} \mathrm{M}$ ) at room temperature (blue line) exhibits a reduction of the main absorption peak at $470 \mathrm{~nm}$ and the appearance of a red-shifted band around $500 \mathrm{~nm}$ as well as a blue-shifted absorption below $400 \mathrm{~nm}$. These spectral features confirm supramolecular aggregation and suggest the formation of H-aggregated CBT units along the supramolecular stacks, as expected for these compounds. ${ }^{21}$

The solid state absorption spectrum (red solid line) that was obtained on thin films resembles that of the aggregated state in solution. The corresponding fluorescence (red dashed line) from the thin film is significantly quenched and red-shifted with a maximum emission intensity at $555 \mathrm{~nm}$ compared to the emission from solution resulting in a Stokes shift of $~ 55 \mathrm{~nm}$. This behavior strongly suggests the formation of H-aggregated CBTs, corresponding to a cofacial 
arrangement of the CBT units in columnar stacks, which is important for efficient charge transport.

$b$ - structural characterization of the bulk and thin films
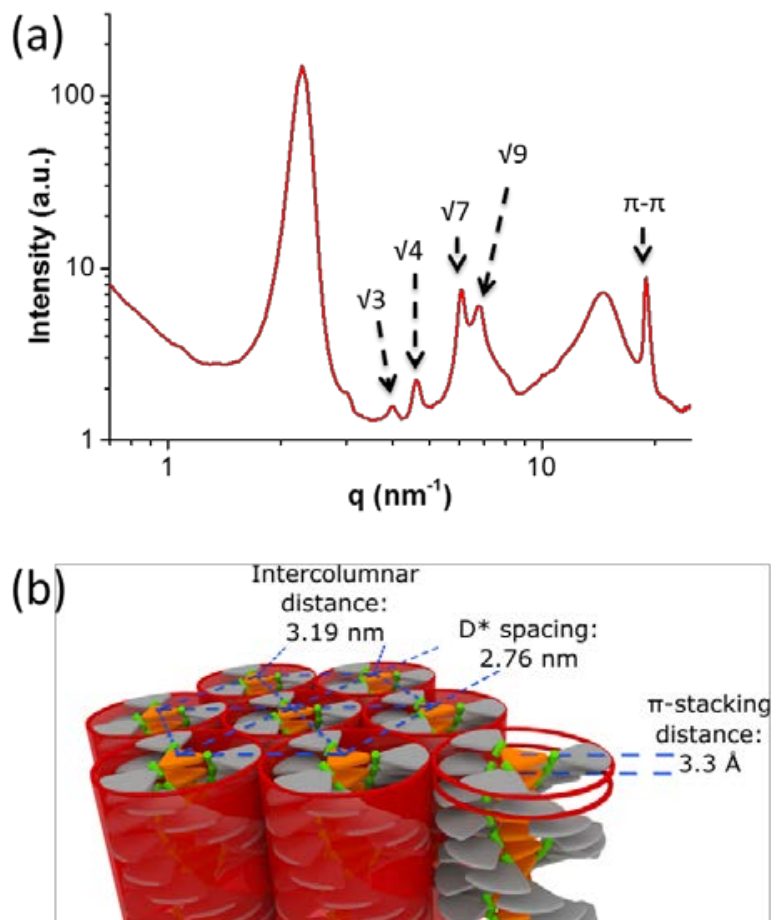

Fig. 2. (a) Small angle X-ray scattering (SAXS) of a powder sample of a-CBT. The locations of the higher order reflections correspond to a columnar hexagonal morphology and the scattering peak for the $\pi-\pi$ stacking of the aromatic cores is indicated. (b) Schematic representation of the arrangement of a-CBT molecules in a columnar hexagonal morphology.

A powder sample and a drop-cast thin film of a-CBT were investigated by small angle X-ray scattering (SAXS), see Fig. 2. Both samples show identical spectra, though the powder sample gave higher intensity due to the limited thickness of the drop-cast film. The SAXS data clearly show columnar hexagonal packing of a-CBT in bulk with a d-spacing of $2.76 \mathrm{~nm}$ corresponding to an intercolumnar distance of $3.19 \mathrm{~nm}$. Furthermore, we see a sharp reflection from the $\pi$ - $\pi$ stacking of a-CBT units corresponding to an interdisc distance of $0.33 \mathrm{~nm}$, which is in good agreement with the highly ordered H-aggregated a-CBT cores as discussed above. 
Differential scanning calorimetry (DSC, Fig. S1) in the range from $20^{\circ} \mathrm{C}$ to $250^{\circ} \mathrm{C}$ does not show any features that could be attributed to phase transitions. Hence, we have no indication of e.g. a ferroelectric and/or liquid crystalline phase. ${ }^{19}$ To obtain a better understanding of the aggregation state in bulk, temperature dependent Fourier transform infrared spectroscopy (FTIR) measurements were performed. The N-H stretching vibration around $3320 \mathrm{~cm}^{-1}$ reveals hydrogen bonds between the amide moieties (Fig. S2). During a heating and cooling cycle from $30{ }^{\circ} \mathrm{C}$ to $215^{\circ} \mathrm{C}$ and back, the $\mathrm{N}-\mathrm{H}$ stretching vibration is slightly shifted to higher energies at elevated temperatures, indicating a weakening of the H-bonds. Similar to the DSC traces no major or sudden changes are observed within this temperature regime that could be associated with a phase transition.

The optical (Fig. 1), structural (Fig. 2) and thermal (Fig. S2) characterizations suggest a significant intercolumnar order due to $\pi$-stacking of the H-aggregated CBT units and hydrogen bonding in drop-casted thin films. We can therefore expect charge transport to mainly take place along the quasi-1D $\pi$-stacks with occasional inter-columnar hopping.

\section{c-electrochemical characterization}

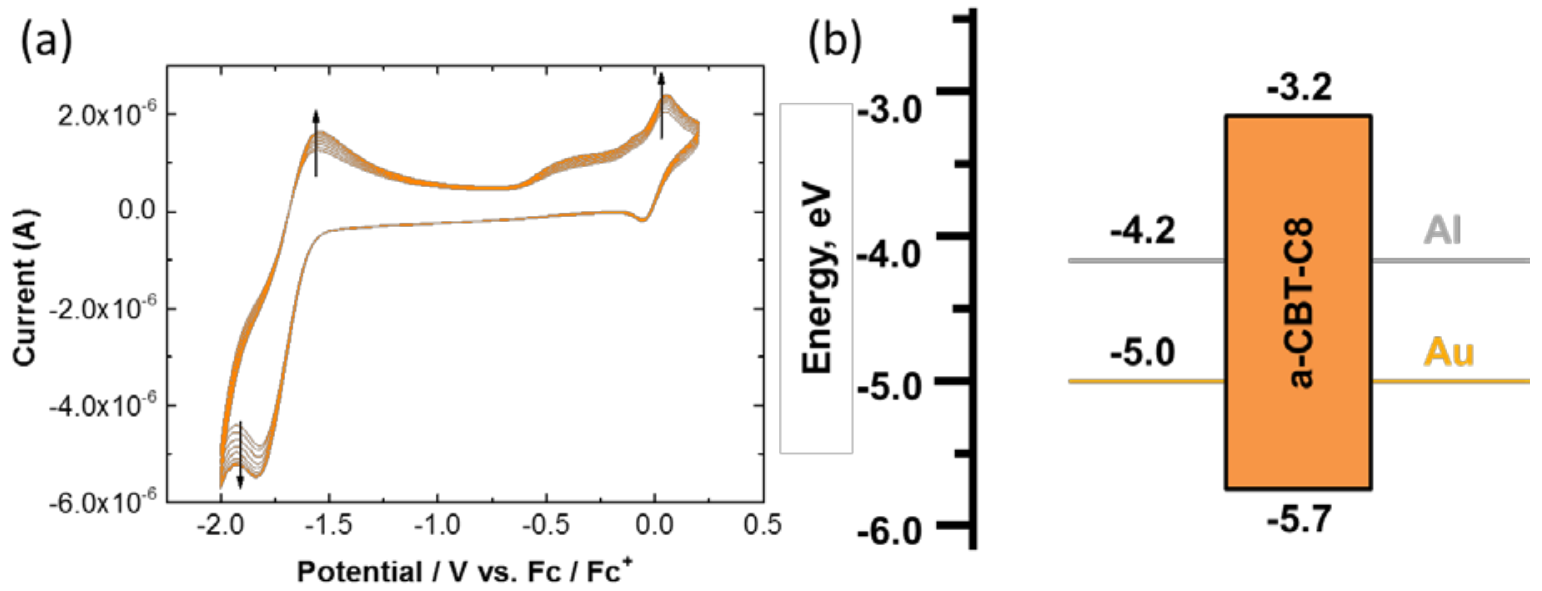

Fig. 3. (a) Cyclic voltammogram of a-CBT in o-DCB with a drop of DMSO, potential vs. ferrocene. (b) Estimated energy level diagram for a-CBT and the approximate work functions of the metal electrodes used in this study. 
From the reduction peak at $-1.6 \mathrm{~V}$ in the $\mathrm{CV}$ measurements vs ferrocene shown in Fig. 3(a) the LUMO of a-CBT could be estimated to be at $-3.2 \mathrm{eV}$. Hence, in order to arrive at an estimate for the a-CBT HOMO energy, we subtract the optical gap determined from the absorption measurements $\sim 2.5 \mathrm{eV}$ (Fig. $1 \mathrm{~b}$ ) from the LUMO energy yielding an estimated HOMO energy of $-5.7 \mathrm{eV}$. Due to the exciton binding energy this resembles a lower limit for the HOMO value.

In Fig. 3(b) the single particle energy levels for a-CBT are shown, together with the approximate work functions of the metal electrodes used in this study. In absence of net interfacial dipoles, hole injection from both $\mathrm{Al}$ electrodes, with a barrier height of $\sim 1.5 \mathrm{eV}$, and Au electrodes, with a barrier height of $\sim 0.7 \mathrm{eV}$, will be significantly hampered. At the same time, the barrier for hole injection from Au is well within the range that can be overcome by ferroelectric-like interfacial dipoles. ${ }^{8,9}$ As explained above, this effect results from the collective behavior of the peripheral amide groups that have previously been shown to enable ferroelectric behavior. ${ }^{19,20}$ In all cases, electron injection and subsequent transport through the material bulk will be hampered by the combination of a large injection barrier and a shallow LUMO level with the associated electron trapping. ${ }^{22}$

\section{$d$-dipolar switching}

The ability of a-CBT to show dipolar switching was tested in simple metal/semiconductor/metal diode devices. In order to observe macroscopic switching behavior in columnar materials, it is necessary that the macro-dipoles, which are parallel to the column axis, ${ }^{19,20}$ are aligned in the direction of the applied electric field, i.e. perpendicular to the electrode's surface. Unfortunately, the columns prefer to align in the plane of the device after deposition, parallel to the electrode’s surface, as shown by atomic force microscopy in Fig. S3 of the SI. Hence, all devices were conditioned by a slowly alternating electric field applied at 
elevated temperatures. Electrical polarization was probed by the double wave method (DWM), which to a large degree allows for the compensation for spurious effects like displacement and leakage currents. For details regarding the device fabrication and testing we refer to the experimental section and the SI section 4, Figs. S4-1 and S4-2. Note, however, that the low sweep speeds used in the DWM to suppress displacement currents do not at all reflect the maximum dipolar switching speed of a-CBT; the latter is beyond the scope of the present work.
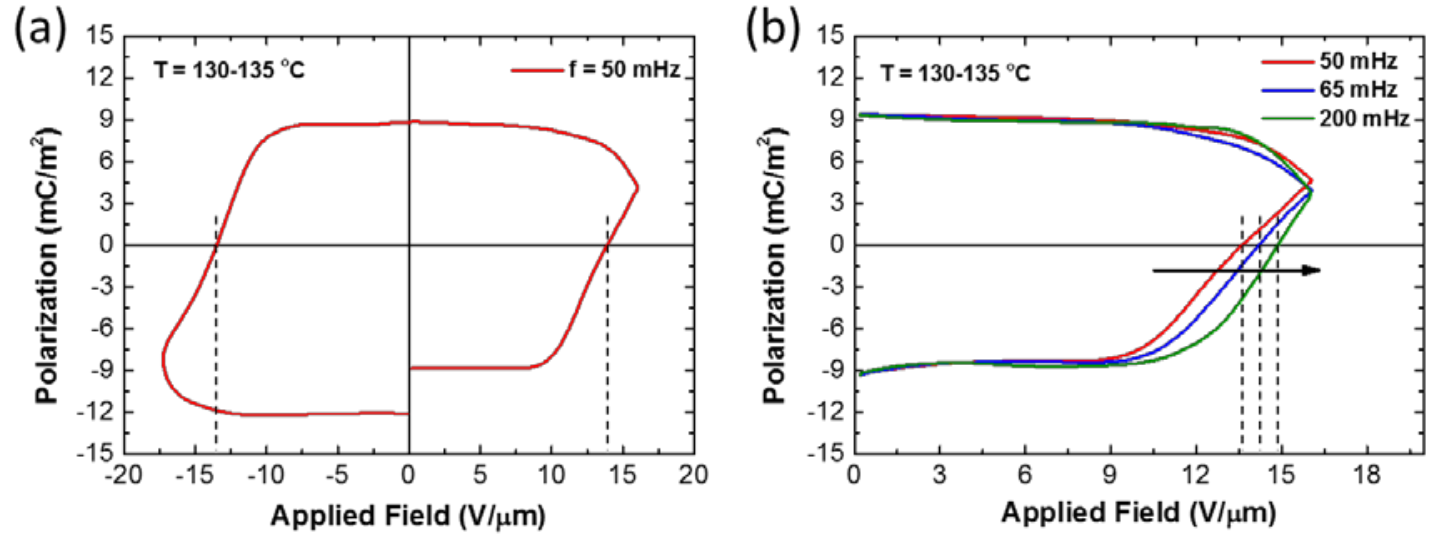

Fig. 4. (a) Ferroelectric-like electrical hysteresis and (b) semi-loops at different switching frequencies obtained on Al/a-CBT/Al devices. Measurements were performed at $\mathrm{T}=130-135$ ${ }^{\circ} \mathrm{C}$. Dashed lines indicate the apparent coercive field $E_{C} \approx 13-14 \mathrm{~V} / \mu \mathrm{m}$ and remnant polarization $P_{r} \approx 9 \mathrm{mC} / \mathrm{m}^{2}$.

The polarization curves in Fig. 4 show the presence of hysteretic dipolar switching in our aCBT diodes. The imperfect closure of the polarization vs. electrical field (PE) curve in panel a) indicates the presence of hysteretic background signals that are caused by effects such as mobile ions or switchable injection currents and that were not completely removed by the DWM. Despite these imperfections, the presence of a well-defined coercive field and remnant polarization clearly indicates a bistable dipolar behavior. ${ }^{23}$ Probing at different frequencies, Fig. 4(b), reveals the characteristic upward shift of $E_{C}$ with increasing frequency. ${ }^{24}$ 
Given the absence of phase transitions in DSC as discussed above, it is unlikely that the observed behavior reflects true bulk ferroelectricity. To further investigate this question, dielectric relaxation spectroscopy (DRS) was performed on micrometer thin films of spincoated a-CBT in a metal/semiconductor/metal configuration. Further details can be found in section 5 of the SI. While molecular rotations are visible in the dielectric spectrum at elevated temperatures $\left(>100{ }^{\circ} \mathrm{C}\right)$, no indication of a bulk ferro-to-paraelectric phase transition was observed (Fig. S6 and S7).

Despite the lack of bulk ferroelectric behavior, the observation of hysteretic polarization loops implies a bistable dipolar switching that is restricted to the two interfacial regions of the metal/a-CBT/metal devices. ${ }^{25}$

The polarity of the interfacial region can be investigated by measuring the local pyroelectric response with a technique called Laser Intensity Modulation Method (LIMM), which has been used to reveal polar depth profiles in numerous (ferroelectric) polymer films. By changing the frequency of modulation, the penetration depth of the thermal wave is tuned to reveal the polarization distribution. The use of this technique to spatially resolve the ferroelectric hysteresis with nanometer resolution in the ferroelectric copolymer $\mathrm{P}(\mathrm{VDF}-\mathrm{TrFE})$ has previously been demonstrated. ${ }^{26}$

Variation of an external electric field at different sample temperatures (Fig. 5) reveals a high electric susceptibility at the metal/a-CBT interface, which is in line with the current hypothesis of interfacial dipolar switching. At $50^{\circ} \mathrm{C}$, a small susceptibility is observed in the first $50 \mathrm{~nm}$ from the electrode interface, with no switching of polarization sign. Only at $150^{\circ} \mathrm{C}$ can the sign of polarization be reversed by an external electric field in the first 40 nanometer. The remaining bulk of the material seems to be incapable of a large paraelectric or ferroelectric response, further suggesting interfacial dipolar switching. However, it is worth noting that for the present purposes, where we aim to modulate a charge injection barrier through a switchable interfacial 
dipole, the absence of true bulk ferroelectricity is inconsequential, as only the first few nm near the injecting contact are important for charge injection.
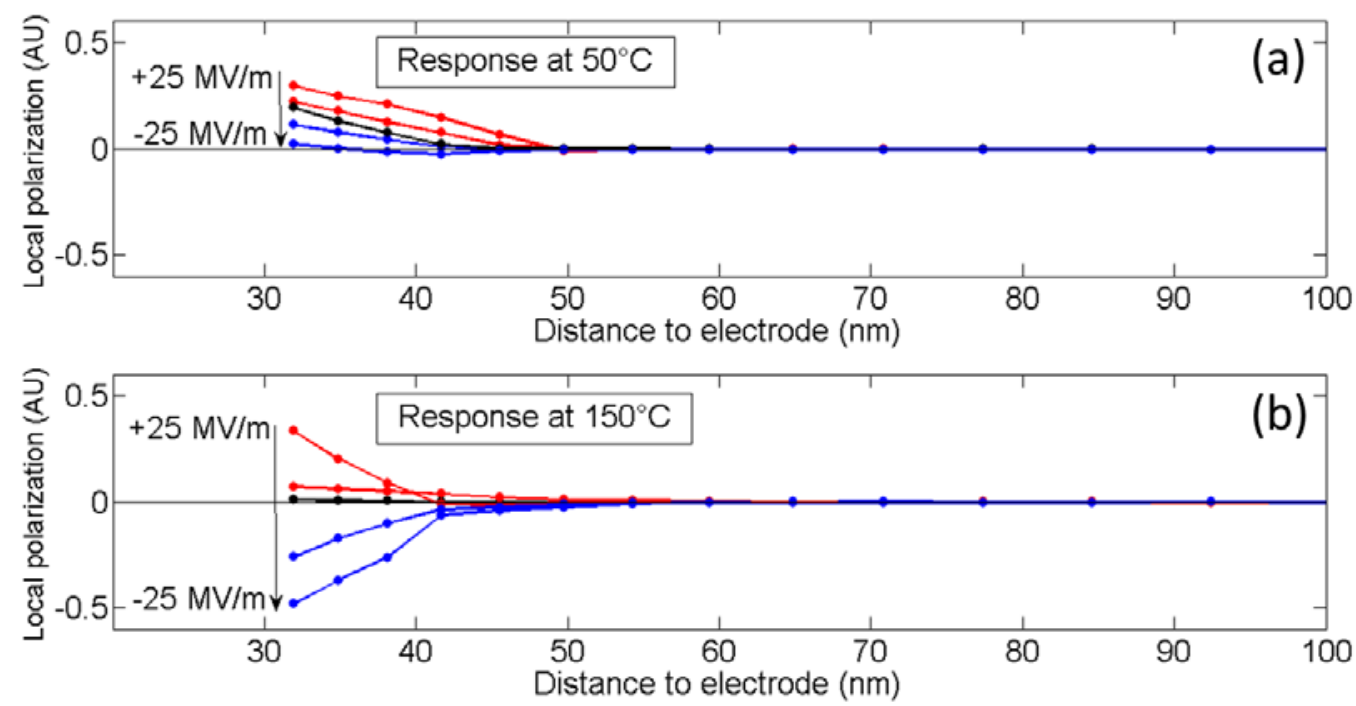

Fig. 5. Pyroelectric depth profiles under external DC bias obtained on an Al/a-CBT/Al device at $\mathrm{T}=50{ }^{\circ} \mathrm{C}$ (a) and $150{ }^{\circ} \mathrm{C}$ (b). The external field is varied from $+25 \mathrm{~V} / \mu \mathrm{m}$ to $-25 \mathrm{~V} / \mu \mathrm{m}$ in steps of $12.5 \mathrm{~V} / \mu \mathrm{m}$.

The effect of the interfacial dipole layer on the hole injection barrier (see Fig. 3) can easily be estimated as

$$
\Delta V \approx \frac{P_{r}}{\varepsilon_{r} \varepsilon_{0}} d_{t y p} \quad, \quad(\text { Eq. } 1)
$$

where $\varepsilon_{0} \varepsilon_{\text {r }}$ is the dielectric constant of the active layer, and $d_{t y p}$ a typical thickness. From DRS, we find that the relative dielectric constant, $\varepsilon_{r}$, of a-CBT is $\varepsilon_{r} \approx 2$. Using $P_{r} \approx 9 \mathrm{mC} / \mathrm{m}^{2}$ and $d_{\text {typ }} \approx 1 \mathrm{~nm}$, we then find that $\Delta V \approx 0.5 \mathrm{eV}$. Under large (positive) forward bias, the interfacial dipole will be directed away from the injecting contact, i.e. with the negative polarization charge facing the contact. Hence, hole injection is facilitated. The original (lower limit for the) hole injection barrier of $\sim 0.7 \mathrm{eV}$ in the case of a Au contact will be reduced to $\sim 0.2 \mathrm{eV}$, which is sufficiently low to enable Ohmic injection. ${ }^{9}$ Using $\mathrm{Al}$ as injecting contact, a 
remaining injection barrier of $\sim 1.0 \mathrm{eV}$ is expected, which will still give rise to injection (or leakage) limited transport.

\section{$e$-electrical characterization}
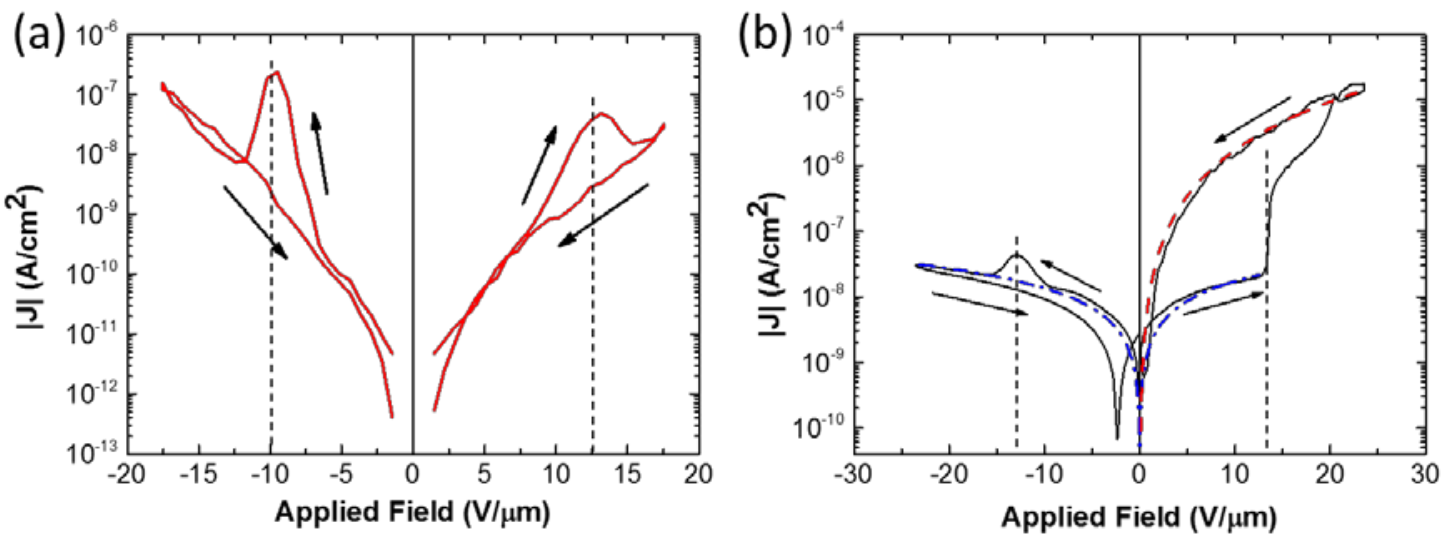

Fig. 6. (a) $j E$ characteristic of an Al/a-CBT/Al device. (b) $j E$ characteristic of an Al/a-CBT/Au device. Arrows indicate sweep directions. Measurements were performed at $\mathrm{T}=130-135{ }^{\circ} \mathrm{C}$. Dashed lines indicate the coercive field $E_{C} \approx 13 \mathrm{~V} / \mu \mathrm{m}$; red dashed and blue dash-dotted lines in (b) indicate fits to space charge limited (Eq. 3) and Ohmic (Eq. 2) conduction with $\mu_{0}=$ $3.7 \cdot 10^{-8} \mathrm{~cm}^{2} / \mathrm{Vs}, \gamma=2.7 \cdot 10^{-3}(\mathrm{~cm} / \mathrm{V})^{1 / 2}$ and $n_{0} \approx 2 \cdot 10^{13} \mathrm{~cm}^{-3}$.

The current vs. field $(j E)$ characteristics for symmetric Al/a-CBT/Al diodes are shown in Fig. 6(a). Coming from large negative (positive) bias, a transient feature in the device current is visible that coincides with the positive (negative) coercive field of $E_{C} \approx 13-14 \mathrm{~V} / \mu \mathrm{m}$, in agreement with Fig. 4. This current peak can be attributed to the displacement current associated with polarization reversal, i.e. $d P / d t$. As the device current itself is mostly linear (dash-dotted line in Fig. 6(b)), it is most likely dominated by leakages associated with background doping, as discussed below. These Ohmic currents obscure the actual injection limited current that is typically highly nonlinear. ${ }^{27}$ Therefore, the interfacial polarization state 
that affects only the injection-limited current but not the background has no visible effect on the device current.

This picture changes dramatically when an asymmetric Al/a-CBT/Au device is measured, see Fig. 6(b). At negative applied field, when hole injection still occurs from the Al contact, again only the transient $d P / d t$ feature is observed. At positive polarity, the Au contact becomes the hole injector. At the coercive field the current now jumps from the Ohmic background value to an on-state current that is two orders of magnitude higher. We carefully checked that this high-current on-state is not a transient phenomenon and that it can be probed multiple times without further changes, as shown in SI section 6. The current in the device is truly bistable. This result is perfectly in line with our prediction on basis of the energy level alignment, see Fig. 3(b). Only by aligning the interfacial dipole moments created by the amide moieties with an (external) electric field can the injection barrier be overcome.

The current switching characteristic in Fig. 6(b) is qualitatively identical to what is observed for the MemOLED devices described in the introduction: upon passing the coercive field, the current jumps from a low, injection- or leakage-limited off-state to a high, space charge limited on-state. ${ }^{4}$ At positive bias, the loop sense is therefore counterclockwise. Importantly, the device shows strong rectification, which is desirable for application in crossbar arrays as it suppresses crosstalk and keeps the switching current low. When (nominally) symmetric Au contacts are used, symmetric $j E$ curves are obtained, exhibiting identical switching behavior at both polarities, see Fig. S8 of the SI. Unavoidably, this compromises rectification.

For the Al/a-CBT/Au device, the $j E$ curves in the off-state can be described by a simple Ohmic conductivity,

$$
j=q \mu_{0} n_{0} V / L
$$

as shown by the blue lines in Fig. 6(b). In the on state, the current density is governed by space charge limited conductivity (SCLC) according to 


$$
j=\frac{9}{8} \varepsilon_{0} \varepsilon_{r} \mu_{0} \frac{V^{2}}{L^{3}} \exp (0.891 \gamma \sqrt{V / L}) .
$$

In Eq. (2,3), $q$ is the elementary charge, $V$ is the applied voltage, $L$ is the layer thickness, $n_{0}$ is the unintentional doping level, $\mu_{0}$ is the zero field mobility and $\gamma$ is a phenomenological parameter quantifying the mobility enhancement by the applied electric field. ${ }^{28}$ The lines in Fig. 6(b) are fits of Eq. (2) and (3) to the $j E$ curve of the Al/a-CBT/Au device, with the mean applied field given by $E=V / L$. The mobility parameters that were obtained by fitting the SCLC regime, $\mu_{0}=3.7 \cdot 10^{-8} \mathrm{~cm}^{2} / \mathrm{Vs}, \gamma=2.7 \cdot 10^{-3}(\mathrm{~cm} / \mathrm{V})^{1 / 2}$, are well in the range expected for organic semiconductors. ${ }^{29,30}$

Using the value for $\mu_{0}$ obtained from the SCLC fit (Eq.3), an estimate for the background charge carrier concentration $n_{0} \approx 2 \cdot 10^{13} \mathrm{~cm}^{-3}$ can be extracted from the linear fit (Eq. 2) to the background current. The presence of a non-negligible background concentration of charge is consistent with the observed nonzero crossings of the $j E$ curves in Fig. 6 and the imperfect shape and closure of the PE curves in Fig. 4. We attribute these phenomena to the presence of slowly moving species, most likely ions. At the same time, these ions can act as countercharge for mobile electrons or holes that give rise to the Ohmic contribution to the total device current. ${ }^{31}$

\section{$f$ - retention}

Finally, we have investigated the retention behavior of our devices. Fig. 7 shows the normalized retention of the on-state current of a $\mathrm{Au} / \mathrm{a}-\mathrm{CBT} / \mathrm{Au}$ device. The retention is measured by poling the diode by a large field $(30 \mathrm{~V} / \mu \mathrm{m})$ and reading, at set points in time, the on-current $j_{\text {on }}$ at a field below the coercive field $\left(E_{r}=10 \mathrm{~V} / \mu \mathrm{m}\right)$. During the waiting time, the device is kept under short-circuit conditions. A mono-exponential decay (dashed line) with a retention time in the order of 5-10 minutes is observed. As the off-state current is time stable, we attribute the falling on-current to a loss in interfacial polarization. Retention times in the 
order of minutes have been observed previously for amide-based organic dipolar materials. ${ }^{20}$ We speculate that the elevated temperature that is needed to enable dipolar switching (130-135 ${ }^{\circ} \mathrm{C}$ ) is also responsible for the limited retention. Unfortunately the retention of the interfacial polarization itself could not be reliably measured in these devices due to the presence of conductive currents that are a few orders of magnitude larger than the (transient) displacement currents associated with polarization reversal.

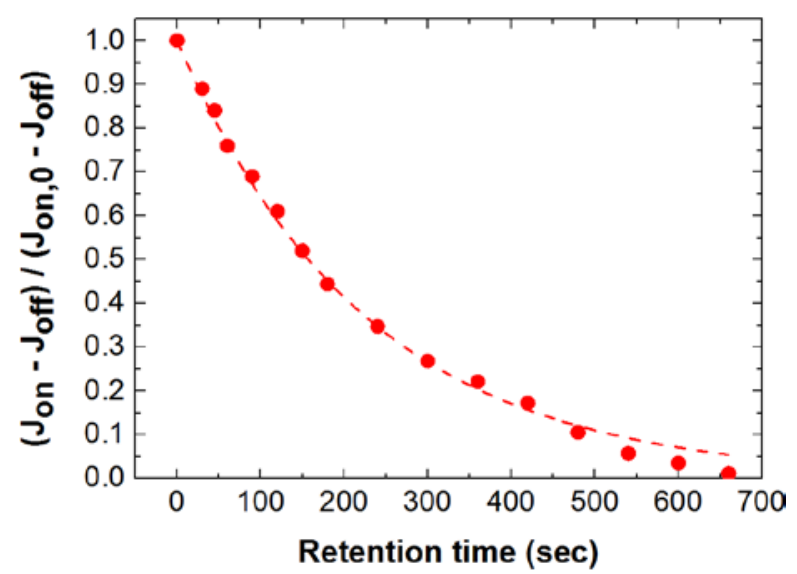

Fig. 7. Retention of the on-current $j_{\text {on }}$ in a Au/a-CBT/Au device measured at $\mathrm{T}=130-135{ }^{\circ} \mathrm{C}$. The current $j_{\text {on }}(t)$ was normalized to its value at time $t=0, j_{\text {on }, 0} \cdot j_{\text {off }}$ is the current density at $t \rightarrow$ $\infty$, i.e. of the depolarized device. Symbols indicate the experiment, the dashed line is a fit to an exponential decay which is typical for relaxation of polar order in molecular materials.

\section{CONCLUSION}

To summarize, we have presented a multifunctional supramolecular organic compound, aCBT, which consists of a semiconducting $\pi$-conjugated core and peripheral dipolar amide side groups. Highly ordered columnar stacks are promoted by $\pi$-stacking of the planar, aromatic carbonyl-bridged triarylamine core and hydrogen bonding of the three amide groups. In thin films, the material organizes in a columnar hexagonal packing. Furthermore, a-CBT was integrated as the active material in simple memory diodes that exhibit both dipolar and resistive 
switching. The dipolar switching could be attributed to field-driven dipole re-orientation near the metal contacts; in contrast, no bulk ferroelectric behavior was observed. The switching of the interfacial dipole layers led to a modulation of the barrier for charge injection from the metal contact. For suitably chosen contact materials, this translated into a switchable device resistance with an on/off ratio of two orders of magnitude and strong rectification.

Combining dipolar and semiconducting properties into a single compound solves many of the problems associated with previously explored memory elements based on phase separated blends of ferroelectric and semiconducting materials. The present results are complementary to recent work in which we demonstrated a polarization-driven modulation of the bulk resistance, as opposed to the contact resistance that is modulated here. ${ }^{32}$ These results are potentially promising for low-cost rewritable memory applications. However, the current need for elevated operation temperatures, and the associated short retention times, will require further attention. The facile processability and tunability by chemical design of organic semiconductors will therefore be a major asset.

\section{EXPERIMENTAL SECTION}

Dimethyl sulfoxide (DMSO) and ortho-dichloro benzene (o-DCB) were used as received. UV-vis measurements were performed on a JASCO V-650 Spectrophotometer, attached with a JASCO ETCR 762 sample holder for solution samples or a JASCO LSE 701 sample holder for thin film samples. Photoluminescence spectra were recorded on a JASCO FP 6500 Spectrofluorometer. For solution measurements, the concentration was adjusted to $5 \cdot 10^{-5} \mathrm{M}$.

Differential scanning calorimetry (DSC) was measured on a DSC Q2000 machine equipped with an auto-sampler and a DSC Refrigerated Cooling System, all from TA Instruments. As vessels Tzero Aluminium Hermetic pans were used. 
Temperature dependent FT-IR data was acquired on a Bruker Tensor 27 equipped with a Pike GladiATR accessory in the temperature range from $30^{\circ} \mathrm{C}$ to $215^{\circ} \mathrm{C}$ with a rate of $10 \mathrm{~K}$ $\min ^{-1}$ upon heating and cooling.

SAXS samples were mounted on V1 grade mica sheets 5-7 $\mu \mathrm{m}$ thick and measured using a SAXSLAB GANESHA system equipped with a GeniX-Cu ultralow divergence source producing X-ray photons with a wavelength of $1.54 \AA$ and a flux of $1 \cdot 10^{8}$ phs $^{-1}$. 2D Scattering patterns were collected using a Pilatus $300 \mathrm{~K}$ silicon pixel detector with a $487 \times 619$ pixel dimension and $172 \mu \mathrm{m}^{2}$ pixel size. The beam center and $q$ range were calibrated using the diffraction peaks of silver behenate. Conversion of 2D images into 1D spectra was accomplished with Saxsgui software.

Cyclic voltammetry was performed under an inert atmosphere with a scan rate of $0.1 \mathrm{~V} \mathrm{~s}^{-1}$ and $1 \mathrm{M}$ tetrabutylammonium hexafluorophosphate in $o$-DCB as the electrolyte. A small amount of DMSO was added to avoid aggregation. The working, counter and reference electrodes were glassy carbon, Pt wire and Ag/AgCl, respectively. The concentration of the sample in the electrolyte was approximately $1 \mathrm{mM}$. All potentials were corrected against $\mathrm{Fc} / \mathrm{FC}^{+}$.

Thin films for absorption/photoluminescence experiments were prepared by drop-casting a solution of $10 \mathrm{mg} / \mathrm{ml}$ (a-CBT in o-DCB/DMSO) on a hot glass slide at $150^{\circ} \mathrm{C}$ and kept at $150^{\circ} \mathrm{C}$ for about 10 min. Thin film capacitor and diode devices were prepared as follows: bottom contacts from gold $(50 \mathrm{~nm})$ or aluminum $(100 \mathrm{~nm})$ were deposited by thermal evaporation under vacuum conditions $\left(\sim 3 \cdot 10^{-7}\right.$ Torr $)$ through a shadow mask on chemically cleaned glass substrates. Prior to Au deposition $5 \mathrm{~nm}$ Cr was deposited to promote adhesion. The organic layer was drop-casted from a warm solution $\left(\sim 90-100{ }^{\circ} \mathrm{C}\right)$ of $20 \mathrm{mg} / \mathrm{ml}$ a-CBT in oDCB/DMSO on a heated substrate $\left(\sim 110^{\circ} \mathrm{C}\right)$ and kept at this temperature for about 10-15 min. At the end of fabrication process, top contacts from aluminum (100 nm) or gold $(50 \mathrm{~nm})$ were 
evaporated through a $90^{\circ}$-rotated shadow mask as described above, giving rise to a cross-bar diode at the intersection of the contacts with a device area between 0.25 and $0.5 \mathrm{~mm}^{2}$. Typical film thicknesses were 0.9-1.2 $\mu \mathrm{m}$, as determined by a Dektak XT profilometer.

Before switching measurements the functional material was conditioned with an electric field in order to align the (interface) dipoles. The electrical alignment consisted of $~ 30$ minutes application of a $0.5 \mathrm{~Hz}$ triangular wave with an amplitude close to coercive field. The positive and negative parts of the triangular wave had equal amplitude but durations that differed by a factor 2. Conditioning was done at an elevated temperature of $\sim 135^{\circ} \mathrm{C}$.

Devices were electrically characterized in the dark using a Janis probe station. Switching signal waveforms were applied by an Agilent 33120a arbitrary waveform generator and amplified by a TReK Model PZD350A high voltage amplifier. The actual circuit current was measured by a Keithley 6485 picoammeter which was visualized and saved via an Agilent DSO7104A oscilloscope. Polarization-field $(P-E)$ hysteresis loops were obtained by integration of the pure switching-current which was obtained as the difference between the full and background responses under quasi-static conditions. This double-wave method (DWM) is described in detail in Refs. ${ }^{33,34}$ For measuring $j E$ curves a low-current Keithley 2636 SMU has been used. Measurement of a full $j E$ curve took typically 20-30 seconds.

Dielectric Relaxation Spectroscopy (DRS) measurements were performed using a Novocontrol nitrogen temperature controller and Alpha-A-analyzer, allowing for temperature control from $-150^{\circ} \mathrm{C}$ to $300{ }^{\circ} \mathrm{C}$ and a frequency range of up to $10 \mathrm{MHz}$. The dielectric response was measured using an AC potential of $100 \mathrm{mV}$ (rms). Samples were prepared as a MIM (metal insulator metal) sandwich by dropcasting of the a-CBT and thermal evaporation of aluminum electrodes.

Laser Intensity Modulation Method (LIMM) measurements used a $10 \mathrm{~mW}$ red Lisa Laser diode laser, modulated by a Tektronix frequency generator, to generate the thermal waves, 
while the pyroelectric response was measured using a Femto messtechnik current amplifier and correlated with a Stanford Research SR-830 RF lock-in amplifier. The frequency range of thermal modulation is $25 \mathrm{kHz}$ to $25 \mathrm{MHz}$.

\section{ASSOCIATED CONTENT}

Supporting Information. Synthesis of a-CBT; DSC and FT-IR spectra of a-CBT; AFM images of drop cast films; Charging curves from DWM; Dielectric relaxation spectroscopy and electrical characterization of Au/a-CBT/Au devices.

\section{AUTHOR INFORMATION}

\section{Corresponding Author}

E-mail: martijn.kemerink@liu.se

\section{ACKNOWLEDGEMENTS}

H.-W.S. and M.Ki. acknowledge financial support from the Bavarian State Ministry of Science, Research, and the Arts for the Collaborative Research Network 'Solar Technologies go Hybrid'. M.Ki. is also thankful for support from the Deutsche Forschungsgemeinschaft (DFG) within SFB 953 "Synthetic Carbon Allotropes". We are also indebted to Doris Hanft for their support in material synthesis. 


\section{REFERENCES}

(1) Scott, J. C. Is There an Immortal Memory? Science 2004, 304 (5667), 62-63.

(2) Scott, J. C.; Bozano, L. D. Nonvolatile Memory Elements Based on Organic Materials. Adv. Mater. 2007, 19 (11), 1452-1463.

(3) Linn, E.; Rosezin, R.; Kügeler, C.; Waser, R. Complementary Resistive Switches for Passive Nanocrossbar Memories. Nat. Mater. 2010, 9 (5), 403-406.

(4) Asadi, K.; de Leeuw, D. M.; de Boer, B.; Blom, P. W. M. Organic Non-Volatile Memories from Ferroelectric Phase-Separated Blends. Nat. Mater. 2008, 7 (7), 547-550.

(5) Heremans, P.; Gelinck, G. H.; Müller, R.; Baeg, K.-J.; Kim, D.-Y.; Noh, Y.-Y. Polymer and Organic Nonvolatile Memory Devices. Chem. Mater. 2011, 23 (3), 341-358.

(6) Li, M.; Stingelin, N.; Michels, J. J.; Spijkman, M.-J.; Asadi, K.; Beerends, R.; Biscarini, F.; Blom, P. W. M.; de Leeuw, D. M. Processing and Low Voltage Switching of Organic Ferroelectric Phase-Separated Bistable Diodes. Adv. Funct. Mater. 2012, 22 (13), 27502757.

(7) Asadi, K.; Wondergem, H. J.; Moghaddam, R. S.; McNeill, C. R.; Stingelin, N.; Noheda, B.; Blom, P. W. M.; de Leeuw, D. M. Spinodal Decomposition of Blends of Semiconducting and Ferroelectric Polymers. Adv. Funct. Mater. 2011, 21 (10), 18871894.

(8) Asadi, K.; de Boer, T. G.; Blom, P. W. M.; de Leeuw, D. M. Tunable Injection Barrier in Organic Resistive Switches Based on Phase-Separated Ferroelectric-Semiconductor Blends. Adv. Funct. Mater. 2009, 19 (19), 3173-3178.

(9) Kemerink, M.; Asadi, K.; Blom, P. W. M.; de Leeuw, D. M. The Operational Mechanism of Ferroelectric-Driven Organic Resistive Switches. Org. Electron. 2012, 13 (1), 147152.

(10) Breemen, A. J. J. M. van; Steen, J.-L. van der; Heck, G. van; Wang, R.; Khikhlovskyi, V.; Kemerink, M.; Gelinck, G. H. Crossbar Arrays of Nonvolatile, Rewritable Polymer Ferroelectric Diode Memories on Plastic Substrates. Appl. Phys. Express 2014, 7 (3), 31602.

(11) Asadi, K.; Li, M.; Stingelin, N.; Blom, P. W. M.; de Leeuw, D. M. Crossbar Memory Array of Organic Bistable Rectifying Diodes for Nonvolatile Data Storage. Appl. Phys. Lett. 2010, 97 (19), 193308.

(12) Khikhlovskyi, V.; Wang, R.; van Breemen, A. J. J. M.; Gelinck, G. H.; Janssen, R. A. J.; Kemerink, M. Nanoscale Organic Ferroelectric Resistive Switches. J. Phys. Chem. C 2014, 118 (6), 3305-3312.

(13) Nougaret, L.; Kassa, H. G.; Cai, R.; Patois, T.; Nysten, B.; van Breemen, A. J. J. M.; Gelinck, G. H.; de Leeuw, D. M.; Marrani, A.; Hu, Z.; Jonas, A. M. Nanoscale Design of Multifunctional Organic Layers for Low-Power High-Density Memory Devices. ACS Nano 2014, 8 (4), 3498-3505.

(14) van Breemen, A.; Zaba, T.; Khikhlovskyi, V.; Michels, J.; Janssen, R.; Kemerink, M.; Gelinck, G. Surface Directed Phase Separation of Semiconductor Ferroelectric Polymer Blends and Their Use in Non-Volatile Memories. Adv. Funct. Mater. 2015, 25 (2), 278286.

(15) Sung, S. H.; Boudouris, B. W. Systematic Control of the Nanostructure of Semiconducting-Ferroelectric Polymer Composites in Thin Film Memory Devices. ACS Macro Lett. 2015, 4 (3), 293-297.

(16) Khikhlovskyi, V.; van Breemen, A. J. J. M.; Janssen, R. A. J.; Gelinck, G. H.; Kemerink, M. Data Retention in Organic Ferroelectric Resistive Switches. Org. Electron. 2016, 31, 56-62. 
(17) Haedler, A. T.; Beyer, S. R.; Hammer, N.; Hildner, R.; Kivala, M.; Köhler, J.; Schmidt, H.-W. Synthesis and Photophysical Properties of Multichromophoric Carbonyl-Bridged Triarylamines. Chem. - Eur. J. 2014, 20 (37), 11708-11718.

(18) Grozema, F. C.; Siebbeles, L. D. A. Mechanism of Charge Transport in Self-Organizing Organic Materials. Int. Rev. Phys. Chem. 2008, 27 (1), 87-138.

(19) Fitié, C. F. C.; Roelofs, W. S. C.; Kemerink, M.; Sijbesma, R. P. Remnant Polarization in Thin Films from a Columnar Liquid Crystal. J. Am. Chem. Soc. 2010, 132 (20), 68926893.

(20) Fitié, C. F. C.; Roelofs, W. S. C.; Magusin, P. C. M. M.; Wübbenhorst, M.; Kemerink, M.; Sijbesma, R. P. Polar Switching in Trialkylbenzene-1,3,5-Tricarboxamides. J. Phys. Chem. B 2012, 116 (13), 3928-3937.

(21) Haedler, A. T.; Kreger, K.; Issac, A.; Wittmann, B.; Kivala, M.; Hammer, N.; Köhler, J.; Schmidt, H.-W.; Hildner, R. Long-Range Energy Transport in Single Supramolecular Nanofibres at Room Temperature. Nature 2015, 523 (7559), 196-199.

(22) Nicolai, H. T.; Kuik, M.; Wetzelaer, G. a. H.; de Boer, B.; Campbell, C.; Risko, C.; Brédas, J. L.; Blom, P. W. M. Unification of Trap-Limited Electron Transport in Semiconducting Polymers. Nat. Mater. 2012, 11 (10), 882-887.

(23) Scott, J. F. Ferroelectrics Go Bananas. J. Phys. Condens. Matter 2008, 20 (2), 21001.

(24) Scott, J. F. Ferroelectric Memories, 1st ed.; Springer Series in Advanced Microelectronics; Springer-Verlag: Berlin Heidelberg, Germany, 2000; Vol. 3.

(25) Nouchi, R.; Tanimoto, T. Substituent-Controlled Reversible Switching of Charge Injection Barrier Heights at Metal/Organic Semiconductor Contacts Modified with Disordered Molecular Monolayers. ACS Nano 2015, 9 (7), 7429-7439.

(26) Putzeys, T.; Wübbenhorst, M. Asymmetric Polarization and Hysteresis Behaviour in Ferroelectric P(VDF-TrFE) (76: 24) Copolymer Thin Films Spatially Resolved via LIMM. Phys Chem Chem Phys 2015, 17 (12), 7767-7774.

(27) van der Holst, J. J. M.; Uijttewaal, M. A.; Ramachandhran, B.; Coehoorn, R.; Bobbert, P. A.; de Wijs, G. A.; de Groot, R. A. Modeling and Analysis of the Three-Dimensional Current Density in Sandwich-Type Single-Carrier Devices of Disordered Organic Semiconductors. Phys. Rev. B 2009, 79 (8), 085203/1-11.

(28) Murgatroyd, P. N. Theory of Space-Charge-Limited Current Enhanced by Frenkel Effect. J. Phys. Appl. Phys. 1970, 3 (2), 151.

(29) Pandey, R.; Gunawan, A. A.; Mkhoyan, K. A.; Holmes, R. J. Efficient Organic Photovoltaic Cells Based on Nanocrystalline Mixtures of Boron Subphthalocyanine Chloride and C60. Adv. Funct. Mater. 2012, 22 (3), 617-624.

(30) Blom, P. W. M.; De Jong, M. J. M.; Van Munster, M. G. Electric-Field and Temperature Dependence of the Hole Mobility in Poly (P-Phenylene Vinylene). Phys. Rev. B 1997, 55 (2), R656.

(31) van Reenen, S.; Matyba, P.; Dzwilewski, A.; Janssen, R. A. J.; Edman, L.; Kemerink, M. A Unifying Model for the Operation of Light-Emitting Electrochemical Cells. J. Am. Chem. Soc. 2010, 132 (39), 13776-13781.

(32) Gorbunov, A. V.; Garcia Iglesias, M.; Guilleme, J.; Roelofs, W. S. C.; Torres, T.; González-Rodríguez, D.; Meijer, E. W.; Kemerink, M. Ferroelectric Self-Assembled Molecular Materials Showing Both Rectifying and Switchable Conductivity. unpublished.

(33) Fukunaga, M.; Noda, Y. New Technique for Measuring Ferroelectric and Antiferroelectric Hysteresis Loops. J. Phys. Soc. Jpn. 2008, 77 (6), 64706.

(34) Khikhlovskyi, V.; Gorbunov, A. V.; van Breemen, A. J. J. M.; Janssen, R. A. J.; Gelinck, G. H.; Kemerink, M. Multi-Bit Organic Ferroelectric Memory. Org. Electron. 2013, 14 (12), 3399-3405. 
Table of Contents graphic

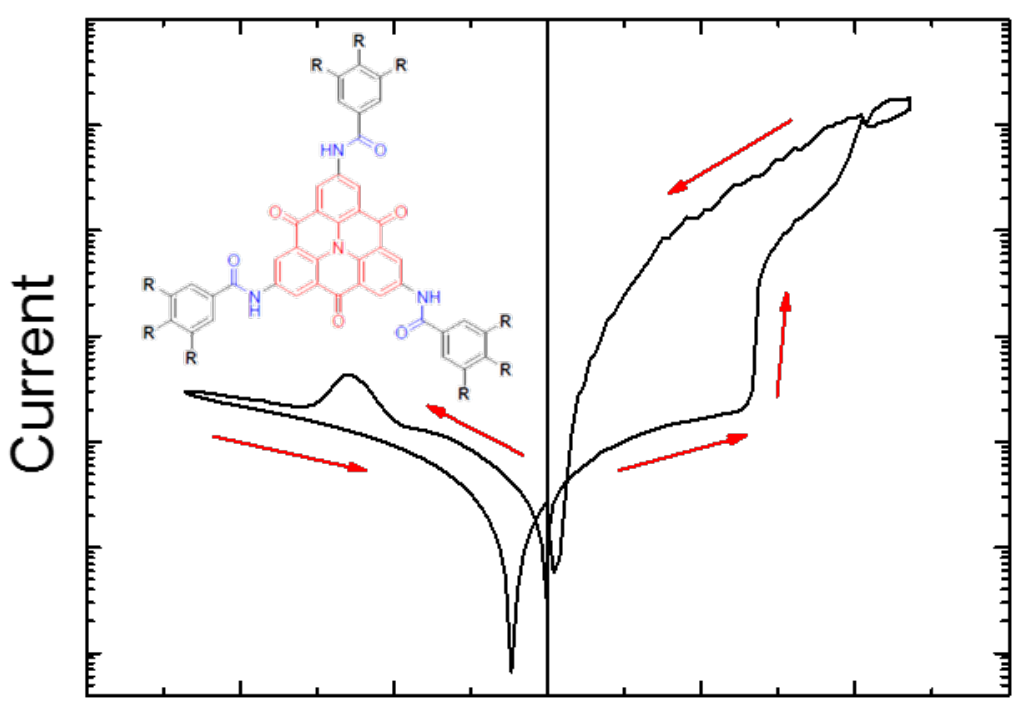

Electric Field 